ANNALES

POLONICI MATHEMATICI

LXXVIII.2 (2002)

\title{
Nullstellensatz and cycles of zeroes of holomorphic mappings
}

\author{
by Ewa Cygan (Kraków)
}

\begin{abstract}
The local Nullstellensatz exponent for holomorphic mappings via intersection theory for the cases of isolated and quasi-complete intersection is considered.

1. Introduction. One of the natural problems in local intersection theory concerns the Nullstellensatz exponent. It is already a classical result that in the case of a set-theoretic complete intersection the degree of the cycle of zeroes of a holomorphic mapping (see $[\mathrm{PT}],[\mathrm{TW}]$ ) is a good exponent for an ideal generated by their components. The general local case seems to be much more difficult; moreover the definition of the cycles of zeroes in this case is quite new. Improper intersections in complex-analytic geometry were first studied by P. Tworzewski $[\mathrm{T}]$. Some interesting applications of the index of intersection for analytic cycles proposed in his paper were found in $[\mathrm{Cy}],[\mathrm{CKT}]$.

Obviously the natural question arises: does this index always give an exponent for Nullstellensatz? This question is also important in algebraic geometry (cf. $[\mathrm{Br}],[\mathrm{JKS}],\left[\mathrm{K}_{1}\right],\left[\mathrm{K}_{2}\right],[\mathrm{Te}]$ ). In this paper we consider two special local cases: the so-called quasi-complete intersection and isolated intersection. The main tool we use to describe the first one is Theorem 3.5 which gives a kind of generalization of Schumacher's Theorem $([€])$. The latter case is treated using only the basic properties of improper isolated intersection presented in $[\mathrm{ATW}]$. One can find another approach to this result in $[\mathrm{S}]$.
\end{abstract}

2. Intersection multiplicity. For the convenience of the reader we compile in this section some basic notions of local intersection theory (see $[\mathrm{Ch}],[\mathrm{D}],[\mathrm{T}]$ for more details).

2000 Mathematics Subject Classification: 32B10, 32B15, 14C17.

Key words and phrases: analytic cycles, intersection theory, Nullstellensatz.

Partially supported by KBN Grant 2 P03A 00115. 
I. Analytic cycles, their multiplicities and proper intersection. In this paper analytic means complex-analytic, and manifold means a complex manifold satisfying the second axiom of countability. Let $M$ be a manifold of dimension $m$. An analytic cycle on $M$ is a formal sum

$$
A=\sum_{j \in J} \alpha_{j} Z_{j}
$$

where $\alpha_{j} \neq 0$ for $j \in J$ are integers and $\left\{Z_{j}\right\}_{j \in J}$ is a locally finite family of distinct irreducible analytic subsets of $M$.

The analytic set $\bigcup_{j \in J} Z_{j}$ is called the support of the cycle $A$ and is denoted by $|A|$. If all the components of $A$ have the same dimension $k$, then $A$ is called a $k$-cycle. We say that $A$ is positive if $\alpha_{j}>0$ for all $j \in J$.

For the cycle $A$, we consider the natural extension of the local multiplicity of analytic sets. Namely, if $a \in M$ and $\nu\left(Z_{j}, a\right)$ denotes the multiplicity of $Z_{j}$ at the point $a$ (see [D, p. 194]), then the sum

$$
\nu(A, a)=\sum_{j \in J} \alpha_{j} \nu\left(Z_{j}, a\right)
$$

is well defined and called the multiplicity of $A$ at $a$.

There exists a unique decomposition

$$
A=T_{(m)}+T_{(m-1)}+\ldots+T_{(0)},
$$

where $T_{(j)}$ is a $j$-cycle for $j=0, \ldots, m$. For our purpose it will be useful to introduce the notion of the extended multiplicity of $A$ at $a$ defined by

$$
\widetilde{\nu}(A, a)=\left(\nu\left(T_{(m)}, a\right), \ldots, \nu\left(T_{(0)}, a\right)\right) \in \mathbb{Z}^{m+1} .
$$

Denote by $\nu(A)$ and $\widetilde{\nu}(A)$ the functions

$$
\nu(A): M \ni x \mapsto \nu(A, x) \in \mathbb{Z}, \quad \widetilde{\nu}(A): M \ni x \mapsto \widetilde{\nu}(A, x) \in \mathbb{Z}^{m+1} .
$$

Observe that $\nu(A, x)=\widetilde{\nu} \widehat{(A, x)}$, where $\widehat{\nu}$ denotes the sum of the coordinates of $\nu \in \mathbb{Z}^{m+1}$.

Let now $X$ and $Y$ be pure dimensional analytic subsets of $M$. We say that $X$ and $Y$ meet properly on $M$ if $\operatorname{dim}(X \cap Y)=\operatorname{dim} X+\operatorname{dim} Y-m$. Then we have the intersection product $X \cdot Y$ of $X$ and $Y$, which is an analytic cycle on $M$ defined by the formula

$$
X \cdot Y=\sum_{Z} i(X \cdot Y, Z) Z,
$$

where the summation extends over all analytic components $Z$ of $X \cap Y$ and $i(X \cdot Y, Z)$ denotes the intersection multiplicity along $Z$ in the sense of Draper ([D, Def. 4.5]; cf. [Ch]). Such multiplicities are positive integers.

II. Intersections of analytic sets with submanifolds. Let $M$ be an $m$ dimensional manifold. Fix a closed $s$-dimensional submanifold $S$ of $M$ and an open subset $U$ of $M$ such that $U \cap S \neq \emptyset$. 
For a given cycle $A=\sum_{j \in J} \alpha_{j} Z_{j}$ analytic on $M$, by its part supported by $S$ we mean the cycle $A^{S}=\sum_{j \in J, Z_{j} \subset S} \alpha_{j} Z_{j}$. Denote by $\mathcal{H}(U)$ the set of all $\mathcal{H}:=\left(H_{1}, \ldots, H_{m-s}\right)$ satisfying the following conditions:

(1) $H_{j}$ is a smooth hypersurface of $U$ containing $U \cap S$ if $j=1, \ldots, m-s$,

(2) $\bigcap_{j=1}^{m-s} T_{x}\left(H_{j}\right)=T_{x} S$ for each $x \in U \cap S$.

For a given analytic subset $Z$ of $M$ of pure dimension $k$ we denote by $\mathcal{H}(U, Z)$ the set of all $\mathcal{H} \in \mathcal{H}(U)$ such that $((U \backslash S) \cap Z) \cap H_{1} \cap \ldots \cap H_{j}$ is an analytic subset of $U \backslash S$ of pure dimension $k-j$ (or empty) for $j=1, \ldots, k$. Each system $\mathcal{H} \in \mathcal{H}(U, Z)$ will be called an admissible system for the set $Z$ and submanifold $S$.

Following [T] we present an algorithm which allows us to produce for every $\mathcal{H} \in \mathcal{H}(U, Z)$ an analytic cycle $Z \cdot \mathcal{H}$ in $S \cap U$. In each step of the algorithm we get a cycle $Z_{i}=Z_{i}^{S}+\left(Z_{i}-Z_{i}^{S}\right)$. Denote by $i_{\mathcal{H}} \in\{0, \ldots, m-s\}$ the first index $i$ for which $\left|Z_{i}-Z_{i}^{S}\right|=\emptyset$.

\section{Algorithm 2.1.}

Step 0 . Let $Z_{0}=Z \cap U$. Then $Z_{0}=Z_{0}^{S}+\left(Z_{0}-Z_{0}^{S}\right)$, where $Z_{0}^{S}$ is the part of $Z_{0}$ supported by $S \cap U$.

Step 1 . Let $Z_{1}=\left(Z_{0}-Z_{0}^{S}\right) \cdot H_{1}$. Then $Z_{1}=Z_{1}^{S}+\left(Z_{1}-Z_{1}^{S}\right)$, where $Z_{1}^{S}$ is the part of $Z_{1}$ supported by $S \cap U$.

Step 2. Let $Z_{2}=\left(Z_{1}-Z_{1}^{S}\right) \cdot H_{2}$. Then $Z_{2}=Z_{2}^{S}+\left(Z_{2}-Z_{2}^{S}\right)$, where $Z_{2}^{S}$ is the part of $Z_{2}$ supported by $S \cap U$. sition

Step $i_{\mathcal{H}}$. Let $Z_{i_{\mathcal{H}}}=\left(Z_{i_{\mathcal{H}}-1}-Z_{i_{\mathcal{H}}-1}^{S}\right) \cdot H_{i_{\mathcal{H}}}$. Now we have the decompo-

$$
Z_{i_{\mathcal{H}}}=Z_{i_{\mathcal{H}}}^{S}+\left(Z_{i_{\mathcal{H}}}-Z_{i_{\mathcal{H}}}^{S}\right), \text { and }\left|Z_{i_{\mathcal{H}}}-Z_{i_{\mathcal{H}}}^{S}\right| \cap S=\emptyset .
$$

We call the positive analytic cycle $Z \cdot \mathcal{H}=Z_{0}^{S}+Z_{1}^{S}+\ldots+Z_{i_{\mathcal{H}}}^{S}$ in $S \cap U$ the result of the above algorithm.

We will say that $Z$ and an admissible system $\mathcal{H}$ realize a quasi-complete intersection at $a$ if every irreducible component of the support of the cycle $Z_{i}-Z_{i}^{S}$ is a complete ideal intersection at $a$ for $i \in\left\{0, \ldots, i_{\mathcal{H}}\right\}$. Recall that an analytic subset $X$ of a manifold $M$ is a complete ideal intersection at $a$ if the ideal of its germ $I\left(X_{a}\right)$ has a system of $p=\operatorname{codim} X_{a}$ generators.

At an arbitrary point $a \in S \cap Z$ we define $g(a)$ as follows. Let

$$
\widetilde{g}(a)=\widetilde{g}(Z, S)(a):=\min _{\operatorname{lex}}\{\widetilde{\nu}(Z \cdot \mathcal{H}, a): \mathcal{H} \in \mathcal{H}(U, Z), a \in U\} \in \mathbb{N}^{s+1}
$$

and $g(a)=g(Z, S)(a)=\widehat{\widetilde{g}(a)}$. The number $g(a)$ is called the index of intersection of $Z$ and $S$ at the point $a$ (see [T, Def. 4.2]). 
Let now $M$ be an open subset of a normed linear space $N$. We are interested in more convenient systems where hypersurfaces have the form of parts of linear hyperplanes from the grassmannian manifold $G^{m-1}(N)$.

Denote by $A(Z, S)(a) \subset\left(G^{m-1}(N)\right)^{m-s}$ the set of all admissible linear systems $\mathcal{H}$ for $Z$ and $S$ at the point $a$ such that $\widetilde{g}(Z, S)(a)=\widetilde{\nu}(Z \cdot \mathcal{H}, a)$.

We will say that $Z$ and $S$ realize a quasi-complete intersection at $a \in$ $Z \cap S$ if there exists a system $\mathcal{H} \in A(Z, S)(a)$ such that $Z$ and $\mathcal{H}$ realize a quasi-complete intersection at $a$.

REMARK $2.2([\mathrm{AR}]$, [N]). If $S$ is a linear subspace of a normed linear space $M$ then to obtain the result of the algorithm it suffices to consider the admissible systems $\mathcal{H}$ which consist of parts of linear hyperplanes. Moreover in such a case the index of intersection of $Z$ and $S$ at $a$ is realized for generic systems of linear hyperplanes in $M$.

III. Intersection of analytic sets-general case. Let $X$ and $Y$ be irreducible analytic subsets of an $m$-dimensional manifold $M$ and let $a \in M$. By standard diagonal construction the multiplicity of intersection of the sets $X$ and $Y$ at $a$ is defined to be

$$
d(a)=d(X, Y)(a)=g\left(X \times Y, \Delta_{M},(a, a)\right) .
$$

The intersection product of the irreducible analytic sets $X$ and $Y$ is the unique analytic cycle $X \bullet Y$ in $M$ such that $\nu(X \bullet Y)=d(X, Y)$ (see [T, Def. 6.3]).

The above definition can be naturally extended to the case of the intersection of a finite number of irreducible analytic subsets and next to arbitrary analytic cycles by multilinearity.

REMARK $2.3([\mathrm{AR}],[\mathrm{N}])$. The index and multiplicity of intersection of an analytic set $X$ with a submanifold $S$ at the point $a$, and of the analytic set $X \times S$ with the diagonal $\Delta_{M}$ at the point $(a, a)$, coincide.

Roughly speaking we are not obliged to pass by the diagonal construction for the case of intersection with a submanifold. This remark will be very useful in our considerations of the cycles of zeroes of holomorphic mappings.

3. Cycles of zeroes of holomorphic mappings and Nullstellensatz. Let $U$ be a neighbourhood of zero in $\mathbb{C}^{m}$ and $\left(f_{1}, \ldots, f_{r}\right): U \rightarrow \mathbb{C}^{r}$ be a holomorphic mapping. Consider two analytic subsets of $U \times \mathbb{C}^{r}$ : the graph of $f, G_{f}=\left\{(x, y) \in U \times \mathbb{C}^{r}: y_{i}=f_{i}(x)\right\}$, and $Y_{f}=U \times\{0\}^{r}$.

Definition 3.1. In this situation we define the cycle $Z_{f}$ on $U$ given by

$$
Z_{f} \times\{0\}=G_{f} \bullet Y_{f}
$$

to be the cycle of zeroes of $f$. 
Obviously $Z_{f}$ is an analytic cycle in $U$ but is not necessarily pure dimensional. Note that if the mapping realizes a set-theoretic complete intersection (which means in particular that $\operatorname{dim} f^{-1}(0)=m-r$ ), then this definition coincides with [TW].

Let us start by recalling the following two results that gave the motivation for this paper. We denote by $I_{z}\left(f_{1}, \ldots, f_{r}\right)$ the ideal of $\mathcal{O}_{z}$ generated by the germs of $f_{i}$ at $z \in U$.

The first theorem is just a special case of [PT, Theorem 1.2].

Theorem 3.2. Suppose that the mapping $\left(f_{1}, \ldots, f_{r}\right): U \rightarrow \mathbb{C}^{r}$ is holomorphic, realizes a set-theoretic complete intersection and $g: U \rightarrow \mathbb{C}$ is a holomorphic function vanishing on the set $f^{-1}(0)$. Let $a \in U$. Then there exist an open neighbourhood $\Omega \subset U$ of a and $h_{1}, \ldots, h_{r} \in \mathcal{O}(\Omega)$ such that

$$
g(z)^{\mu}=f_{1}(z) h_{1}(z)+\ldots+f_{r}(z) h_{r}(z) \quad \text { for } z \in \Omega
$$

where $\mu=\nu\left(Z_{f}, a\right)$ is the degree of the cycle of zeroes of $f$ at a.

The second one is a reformulation of [CKM, Theorem 1].

TheOREM 3.3. Let $f=\left(f_{1}, \ldots, f_{r}\right)$ be a holomorphic mapping in a neighbourhood of $a \in \mathbb{C}^{m}$ realizing a set-theoretic complete intersection at the point a. Then the following conditions are equivalent:

(i) $I_{a}\left(f_{1}, \ldots, f_{r}\right)=\operatorname{Rad} I_{a}\left(f_{1}, \ldots, f_{r}\right)$.

(ii) If $\operatorname{Rad} I_{a}\left(f_{1}, \ldots, f_{r}\right)=I_{1} \cap \ldots \cap I_{s}$, where $I_{j}$ are prime ideals in $\mathcal{O}_{a}$ and $I_{i} \not \subset I_{j}$ for $i \neq j$ then $I_{a}\left(f_{1}, \ldots, f_{r}\right) \mathcal{O}_{I_{j}}=I_{j} \mathcal{O}_{I_{j}}$ for all $j \in\{1, \ldots, s\}$.

In particular, as a direct consequence of Theorem 3.3 (cf. [CKM, Remark 1]) we get the following:

Corollary 3.4. Suppose that $X$ is an $(m-r)$-dimensional analytic subset of a neighbourhood of $a \in \mathbb{C}^{m}$ with the irreducible germ $X_{a}$. If $g=$ $\left(g_{1}, \ldots, g_{r}\right)$ where $g_{1}, \ldots, g_{r}$ are generators of the ideal $I_{a}(X)$ then $\nu(X, a)=$ $\nu\left(Z_{g}, a\right)$.

We start with the following generalization of Schumacher's Theorem (see $[€])$.

TheOREM 3.5. Let $X$ be the zero set of the mapping $\left(f_{1}, \ldots, f_{r}\right): U \rightarrow \mathbb{C}^{r}$ which realizes a set-theoretic complete intersection in $U, g$ be a holomorphic function in $U$ and $a \in X$. Suppose that there exists a dense subset $\widetilde{X}$ of $X$ such that $g_{z} \in I_{z}\left(f_{1}, \ldots, f_{r}\right)$ for $z \in \widetilde{X}$. Then $g_{a} \in I_{a}\left(f_{1}, \ldots, f_{r}\right)$.

Proof. First recall that the family $I_{z}\left(f_{1}, \ldots, f_{r}\right) \subset \mathcal{O}_{z}$ is a coherent family of ideals.

As $\left(f_{1}, \ldots, f_{r}\right)$ realizes a set-theoretic complete intersection in $U$, there exists a neighbourhood $\Omega$ of $a$ such that the primary decomposition of the 
ideal $I_{a}\left(f_{1}, \ldots, f_{r}\right)=J_{a}^{1} \cap \ldots \cap J_{a}^{s}$ corresponds to the irreducible components of the set $X \cap \Omega$, so in $\Omega$ we have:

(1) $X \cap \Omega=X_{1} \cup \ldots \cup X_{s}$,

(2) $I_{z}\left(f_{1}, \ldots, f_{r}\right)=J_{z}^{1} \cap \ldots \cap J_{z}^{s}$ and $J_{z}^{i}$ are primary ideals for $z \in \Omega$,

(3) $I\left(X_{z}^{i}\right)=\operatorname{Rad} J_{z}^{i}$ for $z \in \Omega$ and $i=1, \ldots, s$.

Consider a new coherent family of ideals $J_{z}:=\left\{h_{z} \in \mathcal{O}_{z}: h_{z} g_{z} \in\right.$ $\left.I_{z}\left(f_{1} \ldots, f_{r}\right)\right\}, z \in \Omega$. The coherence implies that the zero set $Z=V\left(\left\{J_{z}\right\}\right)$ of this family is an analytic subset in $\Omega$.

Fix now $i \in\{1, \ldots, s\}$; we will show that $g_{a} \in J_{a}^{i}$. As $\widetilde{X}$ is a dense subset of $X$, by assumption we obtain $X_{a}^{i} \not \subset Z_{a}$ and hence $I\left(Z_{a}\right) \not \subset I\left(X_{a}^{i}\right)$. So there exists $h_{a} \in I\left(Z_{a}\right)$ such that $h_{a} \notin I\left(X_{a}^{i}\right)$. By the Nullstellensatz, $h_{a}^{l} \in J_{a}$ for some $l \in \mathbb{N}$ and as $I\left(X_{i}\right)_{a}$ is a prime ideal, $h_{a}^{l} \notin \operatorname{Rad} J_{a}^{i}=I\left(\left(X_{i}\right)_{a}\right)$.

By definition of $J_{a}$ we have $h_{a}^{l} g_{a} \in I_{a}$ and so $h_{a}^{l} g_{a} \in J_{a}^{i}$. As $J_{a}^{i}$ is a primary ideal we get $g_{a} \in J_{a}^{i}$ and $I_{a}\left(f_{1}, \ldots, f_{r}\right)=J_{a}^{1} \cap \ldots \cap J_{a}^{s}$.

Corollary 3.6. Suppose that $f=\left(f_{1}, \ldots, f_{r}\right)$ realizes a set-theoretic complete intersection in $U$ and $Z_{f}=\sum_{i} k_{i} X_{i}$. Let $g_{i}: U \rightarrow \mathbb{C}$ be a holomorphic function such that $\left.g_{i}\right|_{X_{i}}=0$ for $i=1, \ldots, s$. Then there exists a neighbourhood $W \subset U$ of zero in $\mathbb{C}^{m}$ such that $\prod_{i=1}^{s} g_{i}^{k_{i}}(z)=\sum_{j=1}^{r} h_{j}(z) f_{j}(z)$ for some functions $h_{j}$ holomorphic in $W$.

Proof. Take $h:=g_{1}^{k_{1}} \ldots g_{s}^{k_{s}}$ and note that for each $i=1, \ldots, s$ there exists a dense subset $\widetilde{X}_{i}$ of $X_{i}$ such that $h_{z} \in I_{z}\left(f_{1}, \ldots, f_{r}\right)$ for $z \in \widetilde{X}_{i}$. This is a direct consequence of Theorem 3.2 and the fact that at regular points of $X_{i}$ we have $\nu\left(Z_{f}, z\right)=k_{i}$.

Now Theorem 3.5 implies the result.

From the above corollary we get immediately the following separation type inequality $([\mathrm{Cy}],[\mathrm{CKT}])$ :

REMARK 3.7. (a) In the situation of Theorem 3.5 there exists a neighbourhood of zero in $\mathbb{C}^{m}$ in which

$$
\left|g_{1}(z)\right|^{k_{1}} \ldots\left|g_{s}(z)\right|^{k_{s}} \leq C\left|\left(f_{1}, \ldots, f_{r}\right)(z)\right| \leq c d(z, X)
$$

for some constants $C, c>0$, where $d(\cdot, X)$ denotes the distance to the set $X \subset \mathbb{C}^{m}$.

(b) Working with separation problems one can naturally wonder if a more general inequality with the distance functions could hold:

$$
d\left(z, X_{1}\right)^{k_{1}} \ldots d\left(z, X_{s}\right)^{k_{s}} \leq c\left|\left(f_{1}, \ldots, f_{r}\right)(z)\right|
$$

for some constant $c>0$. Unfortunately, this inequality is false as the example of the function $f(x, y)=x^{3}-y^{2}$ shows.

TheOREM 3.8. Let $f=\left(f_{1}, \ldots, f_{p}\right): U \rightarrow \mathbb{C}^{p}$ be a holomorphic mapping where $U$ is an open neighbourhood of $a \in \mathbb{C}^{n}$. Let $\mathcal{H}=\left(H_{1}, \ldots, H_{p}\right)$ be an 
admissible system of linear hyperplanes for $S=Y_{f}$ at a and suppose that $G_{f}$ and $\mathcal{H}$ realize a quasi-complete intersection at $b=(a, f(a))$. If $g: U \rightarrow \mathbb{C}$ is a holomorphic function vanishing on the zero set of $\left(f_{1}, \ldots, f_{p}\right)$, then $g_{a}^{d} \in I_{a}\left(f_{1}, \ldots, f_{p}\right)$ for $d=\nu\left(G_{f} \cdot \mathcal{H}, b\right)$.

Proof. First note that without loss of generality we can assume that in the neighbourhood $U$ we have $H_{i}=\left\{y_{i}=0\right\}$.

Step (1). By assumption, every component of the cycle $Z_{1}-Z_{1}^{S}=k_{1} Y_{1}+$ $\ldots+k_{r_{1}} Y_{r_{1}}$ is a complete ideal intersection. Let $I_{a}\left(Y_{i}\right)=\left(g_{1, i}\right)$. As $g$ vanishes in a neighbourhood of $a$ on the support of $Z_{1}^{S}$, by Corollary 3.6 we obtain

$$
g_{a}^{\nu\left(Z_{1}^{S}, a\right)} \prod_{i=1}^{r_{1}} g_{1, i}^{k_{i}} \in I_{a}\left(f_{1}\right) .
$$

Step (2). Fix now $i \in\left\{1, \ldots, r_{1}\right\}$ and consider the intersection

$$
Y_{i} \cdot H_{2}=\left(Y_{i} \cdot H_{2}\right)^{S}+Y_{i} \cdot H_{2}-\left(Y_{i} \cdot H_{2}\right)^{S}=\sum_{m=1}^{p_{i, 2}} l_{i, m} X_{i, m}+\sum_{m=1}^{r_{i, 2}} k_{i, m} Y_{i, m} .
$$

Once more we know that each $Y_{i, m}$ is a complete ideal intersection at $a$, so we can write $I_{a}\left(Y_{i, m}\right)=\left(g_{i, m}^{2,1}, g_{i, m}^{2,2}\right)$ for some germs from $\mathcal{O}_{a}$. Applying again Corollary 3.6 and the fact that $g$ vanishes in a neighbourhood of $a$ on the support of the first part of the sum, we get

$$
g_{a}^{\sum_{m=1}^{p_{i, 2}} l_{i, m}} \prod_{m=1}^{r_{i, 2}}\left(g_{i, m}^{2, s}\right)^{k_{i, m}} \in I_{a}\left(g_{1, i}, f_{2}\right)
$$

and as

$$
\sum_{i=1}^{r_{1}} k_{i}\left(\sum_{m=1}^{p_{i, 2}} l_{i, m}\right) \leq \nu\left(Z_{2}^{S}, a\right)
$$

(Corollary 3.4), we have

$$
g_{a}^{\nu\left(Z_{1}^{S}, a\right)+\nu\left(Z_{2}^{S}, a\right)} \prod_{i=1}^{r_{1}} \prod_{m=1}^{r_{i, 2}}\left(g_{i, m}^{2, s}\right)^{k_{i, m} k_{i}} \in I_{a}\left(f_{1}, f_{2}\right)
$$

for $s=1,2$.

It is important to remember that thanks to Corollary 3.4 the generators $g_{1, i}$ do not create any "outer" multiplicity if we take their cycle.

Step $(t)$. By assumption, every component of the cycle $Z_{t-1}-Z_{t-1}^{S}=$ $k_{1}^{t-1} Y_{1}^{t-1}+\ldots+k_{r_{t-1}}^{t-1} Y_{r_{t-1}}^{t-1}$ is a complete ideal intersection. Fix now $i \in$ $\left\{1, \ldots, r_{t-1}\right\}$ and let $I_{a}\left(Y_{i}^{t-1}\right)=\left(g_{i, m}^{t-1,1}, g_{i, m}^{t-1,2}, \ldots, g_{i, m}^{t-1, t-1}\right)$. Consider the 
intersection

$$
\begin{aligned}
Y_{i}^{t-1} \cdot H_{t} & =\left(Y_{i}^{t-1} \cdot H_{t}\right)^{S}+Y_{i}^{t-1} \cdot H_{t}-\left(Y_{i}^{t-1} \cdot H_{t}\right)^{S} \\
& =\sum_{m=1}^{p_{i, t}} l_{i, m}^{t} X_{i, m}^{t}+\sum_{m=1}^{r_{i, t}} k_{i, m}^{t} Y_{i, m}^{t} .
\end{aligned}
$$

Once more we know that each $Y_{i, m}^{t}$ is a complete ideal intersection at $a$, so we can write $I_{a}\left(Y_{i, m}^{t}\right)=\left(g_{i, m}^{t, 1}, g_{i, m}^{t, 2}, \ldots, g_{i, m}^{t, t}\right)$ for some germs from $\mathcal{O}_{a}$. Applying again Corollary 3.6 and the fact that $g$ vanishes in a neighbourhood of $a$ on the support of the first part of the sum, we get

$$
g_{a}^{\sum_{m=1}^{p_{i, t}} l_{i, m}^{t}} \prod_{m=1}^{r_{i, t}}\left(g_{i, m}^{t, s}\right)^{k_{i, m}^{t}} \in I_{a}\left(g_{i, m}^{t-1,1}, g_{i, m}^{t-1,2}, \ldots, g_{i, m}^{t-1, t-1}, f_{t}\right),
$$

and as

$$
\sum_{i=1}^{r_{t-1}} k_{i}^{t-1}\left(\sum_{m=1}^{p_{i, t}} l_{i, m}^{t}\right) \leq \nu\left(Z_{t}^{S}, a\right)
$$

(Corollary 3.4), for $s=1, \ldots, t$ we have

$$
g_{a}^{\nu\left(Z_{1}^{S}, a\right)+\ldots+\nu\left(Z_{t}^{S}, a\right)} \prod_{i=1}^{r_{t}} \prod_{m=1}^{r_{i, t}}\left(g_{i, m}^{t, s}\right)^{k_{i, m}^{t} k_{i}^{t-1}} \in I_{a}\left(f_{1}, \ldots, f_{t}\right) .
$$

By assumption we can repeat the same considerations at each step of the algorithm and finally in the last step we obtain a cycle totally supported by $S$, so $g$ vanishes on this support in a neighbourhood of $a$ and we get

$$
g_{a}^{\nu\left(Z_{1}^{S}, a\right)+\ldots+\nu\left(Z_{p}^{S}, a\right)} \in I_{a}\left(f_{1}, \ldots, f_{p}\right) .
$$

As $\nu\left(Z_{1}^{S}, a\right)+\ldots+\nu\left(Z_{p}^{S}, a\right)=\nu(Z \cdot \mathcal{H}, a)$ this ends the proof.

As a direct consequence of the definition of the cycle of zeroes for a holomorphic mapping we get the following corollary.

Corollary 3.9. Let $f=\left(f_{1}, \ldots, f_{p}\right): U \rightarrow \mathbb{C}^{p}$ be a holomorphic mapping where $U$ is an open neighbourhood of $a \in \mathbb{C}^{n}$. Suppose that $G_{f}$ and $Y_{f}$ realize a quasi-complete intersection at the point $b=(a, f(a))$. If $g: U \rightarrow \mathbb{C}$ is a holomorphic function vanishing on the zero set of $\left(f_{1}, \ldots, f_{p}\right)$, then $g_{a}^{d} \in I_{a}\left(f_{1}, \ldots, f_{p}\right)$ for $d=\nu\left(Z_{f}, a\right)$.

4. Multiplicity of isolated intersection. In the case of an isolated intersection we can use another, equivalent approach to the definition of isolated intersection multiplicity. As in this section we apply the definitions from $[\mathrm{ATW}]$, we first recall basic facts on isolated intersections of analytic sets. For the equivalence of this definition with the general one, see $[\mathrm{T}$, Th. 6.6]. 
Let $Z$ be a pure $k$-dimensional locally analytic subset of a complex manifold $M$ of dimension $m$. Let $N$ be a submanifold of $M$ of dimension $n$ such that $N$ intersects $Z$ at an isolated point $a \in M$. We denote by $\mathcal{F}_{a}(Z, N)$ the set of all locally analytic subsets $V$ of $M$ satisfying:

(1) $V$ has pure dimension $m-k$,

(2) $N_{a} \subset V_{a}$,

(3) $a$ is an isolated point of $V \cap Z$, where $N_{a}, V_{a}$ denote the germs of $N$ and $V$ at $a$.

Observe that for $V \in \mathcal{F}_{a}(Z, N)$ the intersection of $Z$ and $V$ is proper at $a$ and we can consider the classical intersection multiplicity $i(Z \cdot V ; a)$ in the sense of Draper [D] (cf. [W]). We define

$$
\begin{aligned}
\widetilde{i}(Z \cdot N ; a) & =\min \left\{i(Z \cdot V): V \in \mathcal{F}_{a}(Z, N)\right\}, \\
\mathcal{P}_{a}(Z, N) & =\left\{V \in \mathcal{F}_{a}(Z, N): i(Z \cdot V ; a)=\widetilde{i}(Z \cdot N ; a)\right\} .
\end{aligned}
$$

Note that [ATW, Th. 4.4] gives a full characterization of the family $\mathcal{P}_{a}(Z, N)$.

Having disposed of this preliminary step we can now turn to the general case. Let $X, Y$ be pure dimensional locally analytic subsets of a complex manifold $M$ such that $a$ is an isolated point of $X \cap Y$. The positive integer

$$
i(X \cdot Y ; a)=\widetilde{i}\left((X \times Y) \cdot \Delta_{M} ;(a, a)\right)
$$

is defined to be the multiplicity of intersection of $X$ and $Y$ at $a$. It is the same multiplicity as defined in Section 2 [T, Th. 6.6].

If $Y$ is a submanifold the definition of $i(X \cdot Y ; a)$ presented above coincides with that of $\widetilde{i}(X \cdot Y ; a)$ introduced earlier.

Let $f=\left(f_{1}, \ldots, f_{k}\right): U \rightarrow \mathbb{C}^{k}$ be a holomorphic mapping on $U \subset \mathbb{C}^{n}$, $a \in U$ and assume that $f^{-1}(0)=\{a\}$. Define $Z=G_{f}$ to be the graph of $f$ in $M=U \times \mathbb{C}^{k}$ and $N=Y_{f}=U \times\{0\}^{k}$. Suppose that $g$ is a holomorphic function on $U$ such that $g(a)=0$.

THEOREM 4.1 (cf. [S]). In the above situation there exists a neighbourhood $W \subset U$ of the point $a$ in $\mathbb{C}^{n}$ and a system of functions $h_{1}, \ldots, h_{k}$ holomorphic on $W$ such that $g^{d}=f_{1} h_{1}+\ldots+f_{k} h_{k}$ on $W$ where $d=$ $i(Z \cdot N,(a, 0))=\nu\left(Z_{f}, a\right)$.

Proof. Write $c=(a, 0)$ and let $V \in \mathcal{P}_{c}(Z ; N)$. We know that $i(Z \cdot N ; c)=$ $i(Z \cdot V ; c)$, and [ATW, Th. 4.4] implies that $V_{c}$ is a germ of a manifold. We also have $\operatorname{dim} Z=\operatorname{dim} N=n$. As the intersection is isolated it is obvious that $k \geq n$.

Using the standard properties of intersection theory we can assume to have the following situation:

(1) $c=0$ and $M=U \times B \times D \subset \mathbb{C}_{x}^{n} \times \mathbb{C}_{z}^{n} \times \mathbb{C}_{w}^{k-n}$, where $B$ and $D$ are the unit balls in $\mathbb{C}^{n}, \mathbb{C}^{k-n}$ respectively, 
(2) $N=U \times\{0\}^{k}$,

(3) $V=U \times\{0\}^{n} \times D$,

(4) $Z \cap V=\{0\}$,

(5) $\left.\pi\right|_{Z}: Z \rightarrow B$ is an analytic covering of multiplicity $d=i(Z \cdot N ; 0)$, where $\pi: M \rightarrow B$ is the natural projection.

We will now define on $M \times \mathbb{C} \subset \mathbb{C}_{x}^{n} \times \mathbb{C}_{z}^{n} \times \mathbb{C}_{w}^{k-n} \times \mathbb{C}_{t}$ the following function:

$$
P(x, z, w, t)=\prod_{i=1}^{d}\left(t-g\left(x_{i}\right)\right)
$$

for $\left(\left.\pi\right|_{Z}\right)^{-1}(\pi(x, z, w))=\left\{\left(x_{1}, z, w_{1}\right), \ldots,\left(x_{d}, z, w_{d}\right)\right\}$, where $\left(x_{i}, z, w_{i}\right) \in$ $Z$ are counted with their multiplicities. Using standard arguments about symmetric polynomials and the Riemann theorem it is easy to see that $P(x, z, w, t)$ is a holomorphic function.

Observe now that as $N \subset V$ and $(z, w)=\left(y_{1}, \ldots, y_{n}, y_{n+1}, \ldots, y_{k}\right)$, the above function has the following properties:

(1) If $(x, z, w)=\left(x, y_{1}, \ldots, y_{k}\right)=(x, 0)$ then $\left(\left.\pi\right|_{Z}\right)^{-1}(z)=\{0\}$, so we have $P(x, z, w, t)=t^{d}$,

(2) $P(x, f(x), g(x))=0$.

The first property implies that $P(x, z, w, t)-t^{d}$ lies in the ideal generated by $y_{1}, \ldots, y_{k}$, so there exist holomorphic functions $h_{1}, \ldots, h_{k}$ in a neighbourhood $W$ of zero in $M \times \mathbb{C}$ such that $P(x, z, w, t)=t^{d}+y_{1} h_{1}(x, z, w, t)+\ldots+$ $y_{k} h_{k}(x, z, w, t)$ on $W$. Application of the second property ends the proof.

Acknowledgements. The author wishes to thank P. Tworzewski for his helpful remarks and especially the University of Lille 1, where part of this paper was prepared, for the invitation and hospitality.

\section{References}

[AR] R. Achilles and S. Rams, Intersection numbers, Segre numbers and generalized Samuel multiplicities, Arch. Math. (Basel) 77 (2001), 391-398.

[ATW] R. Achilles, P. Tworzewski and T. Winiarski, On improper isolated intersection in complex analytic geometry, Ann. Polon. Math. 51 (1990), 21-36.

[Br] W. D. Brownawell, Bounds for the degree in Nullstellensatz, Ann. of Math. 126 (1987), 577-592.

[CKM] J. Chądzyński, T. Krasiński and A. Miodek, On radical complete intersections, Bull. Polish Acad. Sci. Math. 38 (1990), 1-12.

[Ch] E. M. Chirka, Complex Analytic Sets, Kluwer, 1989.

[Cy] E. Cygan, Intersection theory and separation exponent in complex analytic geometry, Ann. Polon. Math. 69 (1998), 287-299.

[CKT] E. Cygan, T. Krasiński and P. Tworzewski, Separation of algebraic sets and the Łojasiewicz exponent of polynomial mappings, Invent. Math. 136 (1999), 75-87. 
[D] R. Draper, Intersection theory in analytic geometry, Math. Ann. 180 (1969), 175-204.

[JKS] S. Ji, J. Kollár and B. Shiffman, A global Eojasiewicz inequality for algebraic varieties, Trans. Amer. Math. Soc. 329 (1992), 813-818.

[K 1 J. Kollár, Sharp effective Nullstellensatz, J. Amer. Math. Soc. 1 (1988), 963-975.

$\left[\mathrm{K}_{2}\right] \quad$ - Effective Nullstellensatz for arbitrary ideals, J. Eur. Math. Soc. 1 (1999), 317-337.

[€] S. Łojasiewicz, Introduction to Complex Analytic Geometry, Birkhäuser, Basel, 1991.

[N] K. J. Nowak, Improper intersections in complex analytic geometry, Dissertationes Math. 391 (2001).

[PT] A. Płoski and P. Tworzewski, Effective Nullstellensatz on analytic and algebraic varieties, Bull. Polish Acad. Sci. Math. 46 (1998), 31-38.

[R] S. Rams, On intersection product of analytic cycles, Ann. Polon. Math. 73 (2000), 135-146.

[S] S. Spodzieja, Multiplicity and the Eojasiewicz exponent, ibid., 257-267.

[Te] B. Teissier, Résultats récents d'algèbre commutative effective, Séminaire Bourbaki 1989-90 no. 718, Astérisque 189-190 (1990), 107-131.

[T] P. Tworzewski, Intersection theory in complex analytic geometry, Ann. Polon. Math. 62 (1995), 177-191.

[TW] P. Tworzewski and T. Winiarski, Cycles of zeroes of holomorphic mappings, Bull. Polish Acad. Sci. Math. 37 (1989), 95-101.

[W] T. Winiarski, Continuity of total number of intersection, Ann. Polon. Math. 47 (1986), 155-178.

Institute of Mathematics

Jagiellonian University

Reymonta 4

30-059 Kraków, Poland

E-mail: cygan@im.uj.edu.pl 